\section{Oncogenic effects of PAFR ligands produced in tumours upon chemotherapy and radiotherapy}

\section{Roger Chammas, Luciana Nogueira de Sousa Andrade and Sonia Jancar}

The Opinion article from Gabriel Ichim and Stephen W. G. Tait, 'A fate worse than death: apoptosis as an oncogenic process' (Nat. Rev. Cancer 16, 539-548; 2016) ${ }^{1}$, highlights intrinsic mechanisms of failure of commonly used cancer therapies that are heavily dependent on apoptosis. Homeostatic mechanisms are triggered by the presence of dying cells within tissues, favouring the survival of residual tumoural cells in the tumour microenvironment ${ }^{2}$. Understanding these homeostatic mechanisms will provide actionable targets to enhance the effectiveness of therapeutic strategies used in the clinic.

The presence of apoptotic cells with subtumorigenic numbers of mouse melanoma cells promoted tumour engraftment and growth, in a mechanism dependent on the recruitment of inflammatory cells ${ }^{3}$. In a separate study, it was shown that clearance of dying cells by tumour macrophages shifts them towards the protumorigenic M2 phenotype by mechanisms involving the receptor for the lipid mediator platelet-activating factor (PAF), a member of the 1-alkyl,2-acylglycerophosphocholine subclass of lipids 4 .

Lipid mediators are generated in the tumour microenvironment and can modulate tumour growth. Prostaglandin $\mathrm{E}_{2}\left(\mathrm{PGE}_{2}\right)$ is among the most studied molecular effectors of tumour repopulation following chemotherapy ${ }^{5} \cdot \mathrm{PGE}_{2}$ is derived from the metabolism of membrane phospholipids, through the action of phospholipase $\mathrm{A}_{2}$ $\left(\mathrm{PLA}_{2}\right)$. Both $\mathrm{Ca}^{2+}$-dependent PLA $2\left(\mathrm{cPLA}_{2}\right)$ and $\mathrm{Ca}^{2+}$-independent $\mathrm{PLA}_{2}\left(\mathrm{iPLA}_{2}\right.$; also known as PLA2G6) ${ }^{6}$ cleave phosphatidylcholine into arachidonic acid and lysophosphatidylcholine (FIG. 1). Although arachidonic acid can be enzymatically converted into prostanoids, including $\mathrm{PGE}_{2}$, lysophosphatidylcholine is converted into PAF through the action of lysophosphatidylcholine acyltransferases (LPCAT1, LPCAT2, LPCAT3 and LPCAT4) ${ }^{7}$. Therefore, in situations in which $\mathrm{PGE}_{2}$ is produced, depending on the levels of LPCAT expression, PAF is also produced. In a positive feedback loop, through the activation of its receptor (PAFR), PAF induces either activation of $\mathrm{PLA}_{2}$ (REF. 8) or induction of cyclooxygenase 2 (COX2) expression ${ }^{9,10}$, leading to the further release of $\mathrm{PGE}_{2}$. The contribution of PAFR-dependent signalling in tumour repopulation was also demonstrated in its response to chemotherapy and radiotherapy. PAF protects tumour cells from drug-induced cell death, and the administration of PAFR antagonists blocks its protective effect ${ }^{11-13}$.

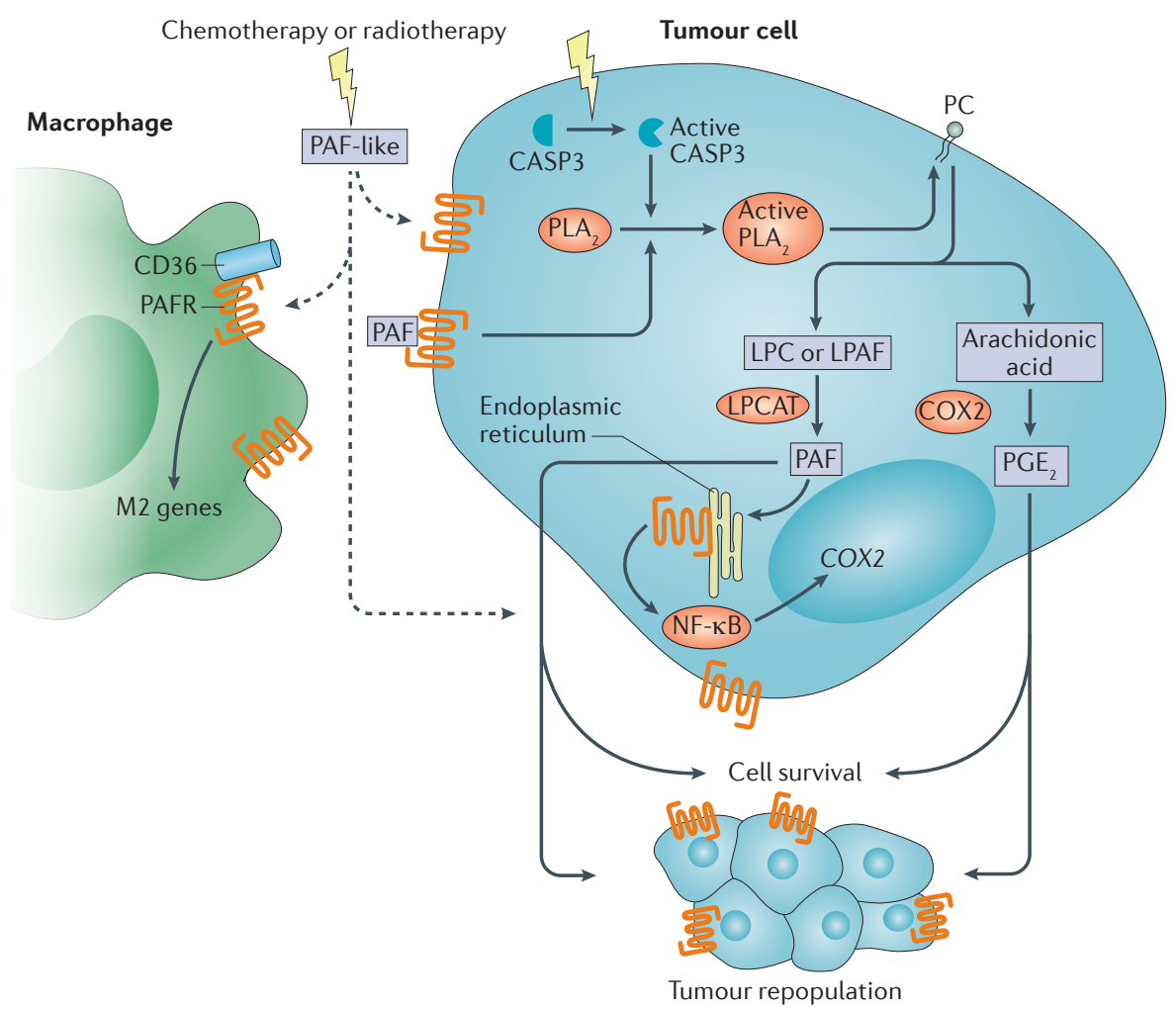

Figure 1 | Pro-oncogenic effects of platelet-activating factor (PAF) and PAF-like molecules after a cell death stimulus. In response to a death stimulus, activated caspase-3 (CASP3) promotes activation of phospholipase $\mathrm{A}_{2}\left(\mathrm{PLA}_{2}\right)$, which cleaves membrane phospholipids such as phosphatidylcholine $(P C)$, releasing arachidonic acid, which can be converted into prostaglandin $E_{2}\left(P E_{2}\right)$ and lysophosphatidylcholine (LPC or lyso-PAF (LPAF)), which can then be converted into PAF through the activity of lysophosphatidylcholine acyltransferase (LPCAT). PAF acts in a positive autocrine loop to further activate $\mathrm{PLA}_{2}$. PAF also interacts with intracellular PAF receptor (PAFR), activating nuclear factor- $\kappa \mathrm{B}$ (NF- $\kappa B$ ), which in turn induces cyclooxygenase 2 (COX2) transcription, thus potentiating $\mathrm{PGE}_{2}$ production. Both PAF and $\mathrm{PGE}_{2}$ act as pro-survival stimuli in tumour cells, promoting proliferation of the surviving cells and leading to tumour repopulation. Activation of PAFR in macrophages promotes reprogramming towards an anti-inflammatory and pro-tumorigenic profile (M2 genes). Moreover, irradiation and chemotherapy generate a wide range of PAFR ligands (PAF-like) in the tumour microenvironment that act as PAFR agonists (dashed arrows), amplifying the effect of PAFR activation in tumour cells and macrophages. 


\section{CORRESPONDENCE}

developed in the past, but none has yet been tested for clinical use in cancer. PAFR antagonists are likely to complement strategies that target tumour-associated macrophages (colony-stimulating factor 1 receptor (CSF1R) antagonists) and $\mathrm{PGE}_{2}$ (COX2 inhibitors), which, together, will improve the efficacy of apoptogenic therapies.

Roger Chammas and Luciana Nogueira de Sousa Andrade are at the Instituto do Câncer do Estado de São Paulo, Faculdade de Medicina da Universidade de São Paulo, São Paulo, Brazil.

Sonia Jancar is at the Instituto de Ciências Biomédicas da Universidade de São Paulo, São Paulo, Brazil

Correspondence to R.C.rchammas@usp.br doi:10.1038/nrc.2017.15 Published online 10 Mar 2017

\section{Acknowledgements}

Funded by Fundação de Amparo à Pesquisa do Estado de São Paulo (FAPESP, 2013/15719-0 and 2015/22814-5).

\section{Competing interests statement}

The authors declare no competing interests.

1. Ichim, G. \& Tait, S. W. G. A fate worse than death apoptosis as an oncogenic process. Nat. Rev. Cancer 16, 539-548 (2016)

2. Huang, Q. et al. Caspase 3-mediated stimulation of tumor cell repopulation during cancer radiotherapy. Nat. Med. 17, 860-866 (2011).

3. Correa, M. et al. Transient inflammatory response induced by apoptotic cells is an important mediator of melanoma cell engraftment and growth. Int. J. Cancer 114 356-363 (2005).
4. Ferracini, M. Rios, F J O Pecenin, M \& Jancar S. Clearance of apoptotic cells by macrophages induces regulatory phenotype and involves stimulation of CD36 and PAFR receptor. Mediators Inflamm. 2013 950273 (2013).

5. Kurtova, A. V. et al. Blocking $\mathrm{PGE}_{2}$-induced tumour repopulation abrogates bladder cancer chemoresistance. Nature 517, 209-213 (2015).

6. McHowat, J. et al. Platelet-activating factor and metastasis: calcium-independent phospholipase $A_{2} \beta-$ deficiency protects against breast cancer metastasis to the lung. Am. J. Physiol. Cell Physiol. 300 C825-C832 (2011)

7. Murakami, M. \& Kudo, I. Recent advances in molecular biology and physiology of the prostaglandin $\mathrm{E}_{2}$-biosynthetic pathway. Prog. Lipid Res. 43, 3-35 (2004)

8. Oestvang, J., Anthonsen, M. W. \& Johansen, B. LysoPC and PAF trigger arachidonic acid release by divergent signaling mechanisms in monocytes. J. Lipids 2011, 532145 (2011)

9. Glaser, K. B., Asmis, R. \& Dennis, E. A. PAF receptor mediated PGE2 production in LPS primed P388D1 macrophage-like cells. Adv Prostaglandin Thromboxane Leukot Res. 21 A, 249-255 (1991).

10. Ishizuka, E. K., Filgueiras, L. R., Rios, F. J. Serezani, C. H. \& Jancar, S. PAFR activation of NF-кB p65 or p105 precursor dictates pro- and antiinflammatory responses during TLR activation in murine macrophages. Sci. Rep. 6, 16 (2092).

11. Onuchic, A. C. et al. Expression of PAFR as part of a prosurvival response to chemotherapy: a novel target for combination therapy in melanoma. Mediators Inflamm. 2012, 175408 (2012)

12. Sahu, R. P. et al. Chemotherapeutic agents subvert tumor immunity by generating agonists of plateletactivating factor. Cancer Res. 74, 7069-7078 (2014).

13. Sahu, R. P., Ferracini, M. \& Travers, J. B. Systemic chemotherapy is modulated by platelet-activating factorreceptor agonists. Mediators Inflamm. 2015, 820543 (2015).

14. Sahu, R. P. et al. Radiation therapy generates plateletactivating factor agonists. Oncotarget 7 , 20788-20800 (2016) 